\title{
Umbral Calculus and the Frobenius-Euler Polynomials
}

\author{
Dae San Kim, ${ }^{1}$ Taekyun Kim, ${ }^{2}$ and Sang-Hun Lee ${ }^{3}$ \\ ${ }^{1}$ Department of Mathematics, Sogang University, Seoul 121-742, Republic of Korea \\ ${ }^{2}$ Department of Mathematics, Kwangwoon University, Seoul 139-701, Republic of Korea \\ ${ }^{3}$ Division of General Education, Kwangwoon University, Seoul 139-701, Republic of Korea \\ Correspondence should be addressed to Taekyun Kim; tkkim@kw.ac.kr
}

Received 27 November 2012; Accepted 19 December 2012

Academic Editor: Juan J. Trujillo

Copyright (C) 2013 Dae San Kim et al. This is an open access article distributed under the Creative Commons Attribution License, which permits unrestricted use, distribution, and reproduction in any medium, provided the original work is properly cited.

We study some properties of umbral calculus related to the Appell sequence. From those properties, we derive new and interesting identities of the Frobenius-Euler polynomials.

\section{Introduction}

Let $\mathbf{C}$ be the complex number field. For $\lambda \in \mathbf{C}$ with $\lambda \neq 1$, the Frobenius-Euler polynomials are defined by the generating function to be

$$
\frac{1-\lambda}{e^{t}-\lambda} e^{x t}=e^{H(x \mid \lambda) t}=\sum_{n=0}^{\infty} H_{n}(x \mid \lambda) \frac{t^{n}}{n !}
$$

(see [1-5]) with the usual convention about replacing $H^{n}(x \mid$ $\lambda)$ by $H_{n}(x \mid \lambda)$.

In the special case, $x=0, H_{n}(0 \mid \lambda)=H_{n}(\lambda)$ are called the $n$th Frobenius-Euler numbers. By (1), we get

$$
H_{n}(x \mid \lambda)=\sum_{l=0}^{n}\left(\begin{array}{l}
n \\
l
\end{array}\right) H_{n-l}(\lambda) x^{l}=(H(\lambda)+x)^{n},
$$

(see [6-9]) with the usual convention about replacing $H^{n}(\lambda)$ by $H_{n}(\lambda)$.

Thus, from (1) and (2), we note that

$$
(H(\lambda)+1)^{n}-\lambda H_{n}(\lambda)=(1-\lambda) \delta_{0, n},
$$

where $\delta_{n, k}$ is the kronecker symbol (see $\left.[1,10,11]\right)$.
For $r \in \mathbf{Z}_{+}$, the Frobenius-Euler polynomials of order $r$ are defined by the generating function to be

$$
\begin{aligned}
\left(\frac{1-\lambda}{e^{t}-\lambda}\right)^{r} e^{x t} & =\underbrace{\left(\frac{1-\lambda}{e^{t}-\lambda}\right) \times \cdots \times\left(\frac{1-\lambda}{e^{t}-\lambda}\right)}_{r \text {-times }} e^{x t} \\
& =\sum_{n=0}^{\infty} H_{n}^{(r)}(x \mid \lambda) \frac{t^{n}}{n !} .
\end{aligned}
$$

In the special case, $x=0, H_{n}^{(r)}(0 \mid \lambda)=H_{n}^{(r)}(\lambda)$ are called the $n$th Frobenius-Euler numbers of order $r$ (see $[1,10])$.

From (4), we can derive the following equation:

$$
\begin{gathered}
H_{n}^{(r)}(x \mid \lambda)=\sum_{l=0}^{n}\left(\begin{array}{l}
n \\
l
\end{array}\right) H_{n-l}^{(r)}(\lambda) x^{l}, \\
H_{n}^{(r)}(\lambda)=\sum_{l_{1}+\cdots+l_{r}=n}\left(\begin{array}{c}
n \\
l_{1}, \ldots, l_{r}
\end{array}\right) H_{l_{1}}(\lambda) \cdots H_{l_{r}}(\lambda) .
\end{gathered}
$$

By (5), we see that $H_{n}^{(r)}(x \mid \lambda)$ is a monic polynomial of degree $n$ with coefficients in $\mathbf{Q}(\lambda)$.

Let $\mathbb{P}$ be the algebra of polynomials in the single variable $x$ over $\mathbf{C}$ and let $\mathbb{P}^{*}$ be the vector space of all linear functionals on $\mathbb{P}$. As is known, $\langle L \mid p(x)\rangle$ denotes the action of the linear functional $L$ on a polynomial $p(x)$ and we remind that 
the addition and scalar multiplication on $\mathbb{P}^{*}$ are, respectively, defined by

$$
\begin{gathered}
\langle L+M \mid p(x)\rangle=\langle L \mid p(x)\rangle+\langle M \mid p(x)\rangle, \\
\langle c L \mid p(x)\rangle=c\langle L \mid p(x)\rangle,
\end{gathered}
$$

where $c$ is a complex constant (see $[3,12])$.

Let $\mathbf{F}$ denote the algebra of formal power series:

$$
\mathbf{F}=\left\{f(t)=\sum_{k=0}^{\infty} \frac{a_{k}}{k !} t^{k} \mid a_{k} \in \mathbf{C}\right\}
$$

(see $[3,12])$. The formal power series define a linear functional on $\mathbb{P}$ by setting

$$
\left\langle f(t) \mid x^{n}\right\rangle=a_{n}, \quad \forall n \geq 0 .
$$

Indeed, by (7) and (8), we get

$$
\left\langle t^{k} \mid x^{n}\right\rangle=n ! \delta_{n, k} \quad(n, k \geq 0)
$$

$($ see $[3,12])$. This kind of algebra is called an umbral algebra.

The order $O(f(t))$ of a nonzero power series $f(t)$ is the smallest integer $k$ for which the coefficient of $t^{k}$ does not vanish. A series $f(t)$ for which $O(f(t))=1$ is said to be an invertible series (see $[2,12]$ ). For $f(t), g(t) \in \mathbf{F}$, and $p(x) \in \mathbb{P}$, we have

$$
\begin{aligned}
\langle f(t) g(t) \mid p(x)\rangle & =\langle f(t) \mid g(t) p(x)\rangle \\
& =\langle g(t) \mid f(t) p(x)\rangle
\end{aligned}
$$

(see [12]). One should keep in mind that each $f(t) \in \mathbf{F}$ plays three roles in the umbral calculus: a formal power series, a linear functional, and a linear operator. To illustrate this, let $p(x) \in \mathbb{P}$ and $f(t)=e^{y t} \in \mathbf{F}$. As a linear functional, $e^{y t}$ satisfies $\left\langle e^{y t} \mid p(x)\right\rangle=p(y)$. As a linear operator, $e^{y t}$ satisfies $e^{y t} p(x)=p(x+y)$ (see [12]). Let $s_{n}(x)$ denote a polynomial in $x$ with degree $n$. Let us assume that $f(t)$ is a delta series and $g(t)$ is an invertible series. Then there exists a unique sequence $s_{n}(x)$ of polynomials such that $\left\langle g(t) f(t)^{k} \mid s_{n}(x)\right\rangle=$ $n ! \delta_{n, k}$ for all $n, k \geq 0$ (see $\left.[3,12]\right)$. This sequence $s_{n}(x)$ is called the Sheffer sequence for $(g(t), f(t))$ which is denoted by $s_{n}(x) \sim(g(t), f(t))$. If $s_{n}(x) \sim(1, f(t))$, then $s_{n}(x)$ is called the associated sequence for $f(t)$. If $s_{n}(x) \sim(g(t), t)$, then $s_{n}(x)$ is called the Appell sequence.

Let $s_{n}(x) \sim(g(t), f(t))$. Then we see that

$$
\begin{aligned}
& h(t)= \sum_{k=0}^{\infty} \frac{\left\langle h(t) \mid s_{k}(x)\right\rangle}{k !} g(t) f(t)^{k}, \quad h(t) \in \mathbf{F}, \\
& p(x)= \sum_{k=0}^{\infty} \frac{\left\langle g(t) f(t)^{k} \mid p(x)\right\rangle}{k !} s_{k}(x), \quad p(x) \in \mathbb{P}, \\
& f(t) s_{n}(x)=n s_{n-1}(x), \\
& \\
& \frac{\langle f(t) \mid p(\alpha x)\rangle=\langle f(\alpha t|p(x)\rangle,}{g(\bar{f}(t))} e^{y \bar{f}(t)}=\sum_{k=0}^{\infty} \frac{s_{k}(y)}{k !} t^{k}, \quad \forall y \in \mathbf{C},
\end{aligned}
$$

where $\bar{f}(t)$ is the compositional inverse of $f(t)$ (see [3]). In this paper, we study some properties of umbral calculus related to the Appell sequence. For those properties, we derive new and interesting identities of the Frobenius-Euler polynomials.

\section{The Frobenius-Euler Polynomials and Umbral Calculus}

By (4) and (12), we see that

$$
H_{n}^{(r)}(x \mid \lambda) \sim\left(\left(\frac{e^{t}-\lambda}{1-\lambda}\right)^{r}, t\right) .
$$

Thus, by (13), we get

$$
\left\langle\left(\frac{e^{t}-\lambda}{1-\lambda}\right)^{r} t^{k} \mid H_{n}^{(r)}(x \mid \lambda)\right\rangle=n ! \delta_{n, k} .
$$

Let

$$
\mathbb{P}_{n}(\lambda)=\{p(x) \in \mathbf{Q}(\lambda)[x] \mid \operatorname{deg} p(x) \leq n\} .
$$

Then it is an $(n+1)$-dimensional vector space over $\mathbf{Q}(\lambda)$.

So we see that $\left\{H_{0}^{(r)}(x \mid \lambda), H_{1}^{(r)}(x \mid \lambda), \ldots, H_{n}^{(r)}(x \mid \lambda)\right\}$ is a basis for $\mathbb{P}_{n}(\lambda)$. For $p(x) \in \mathbb{P}_{n}(\lambda)$, let

$$
p(x)=\sum_{k=0}^{n} C_{k} H_{k}^{(r)}(x \mid \lambda), \quad(n \geq 0) .
$$

Then, by (13), (14), and (16), we get

$$
\begin{aligned}
& \left\langle\left(\frac{e^{t}-\lambda}{1-\lambda}\right)^{r} t^{k} \mid p(x)\right\rangle \\
& =\sum_{l=0}^{n} C_{l}\left\langle\left(\frac{e^{t}-\lambda}{1-\lambda}\right)^{r} t^{k} \mid H_{l}^{(r)}(x \mid \lambda)\right\rangle \\
& =\sum_{l=0}^{n} C_{l} l ! \delta_{l, k}=k ! C_{k} .
\end{aligned}
$$

From (17), we have

$$
\begin{aligned}
C_{k} & =\frac{1}{k !}\left\langle\left(\frac{e^{t}-\lambda}{1-\lambda}\right)^{r} t^{k} \mid p(x)\right\rangle \\
& =\frac{1}{k !}\left\langle\left(\frac{e^{t}-\lambda}{1-\lambda}\right)^{r} \mid D^{k} p(x)\right\rangle \\
& =\frac{1}{k !(1-\lambda)^{r}} \sum_{j=0}^{r}\left(\begin{array}{l}
r \\
j
\end{array}\right)(-\lambda)^{r-j}\left\langle e^{j t} \mid D^{k} p(x)\right\rangle \\
& =\frac{1}{k !(1-\lambda)^{r}} \sum_{j=0}^{r}\left(\begin{array}{l}
r \\
j
\end{array}\right)(-\lambda)^{r-j}\left\langle t^{0} \mid e^{j t} D^{k} p(x)\right\rangle \\
& =\frac{1}{k !(1-\lambda)^{r}} \sum_{j=0}^{r}\left(\begin{array}{l}
r \\
j
\end{array}\right)(-\lambda)^{r-j}\left\langle t^{0} \mid D^{k} p(x+j)\right\rangle .
\end{aligned}
$$

Therefore, by (16) and (18), we obtain the following theorem. 
Theorem 1. For $p(x) \in \mathbb{P}_{n}(\lambda)$, let

$$
p(x)=\sum_{k=0}^{n} C_{k} H_{k}^{(r)}(x) .
$$

Then one has

$$
C_{k}=\frac{1}{k !(1-\lambda)^{r}} \sum_{j=0}^{r}\left(\begin{array}{l}
r \\
j
\end{array}\right)(-\lambda)^{r-j} D^{k} p(j),
$$

where $D p(x)=d p(x) / d x$.

From Theorem 1, we note that

$$
\begin{aligned}
p(x)= & \frac{1}{(1-\lambda)^{r}} \\
& \cdot \sum_{k=0}^{n}\left\{\sum_{j=0}^{r} \frac{1}{k !}\left(\begin{array}{l}
r \\
j
\end{array}\right)(-\lambda)^{r-j} D^{k} p(j)\right\} H_{k}^{(r)}(x \mid \lambda) .
\end{aligned}
$$

Let us consider the operator $\widetilde{\Delta}_{\lambda}$ with $\widetilde{\Delta}_{\lambda} f(x)=f(x+1)-$ $\lambda f(x)$ and let $J_{\lambda}=(1 /(1-\lambda)) \widetilde{\Delta}_{\lambda}$. Then we have

$$
J_{\lambda}(f)(x)=\frac{1}{1-\lambda}\{f(x+1)-\lambda f(x)\} .
$$

Thus, by (22), we get

$$
J_{\lambda}\left(H_{n}^{(r)}(x \mid \lambda)\right)=\frac{1}{1-\lambda}\left\{H_{n}^{(r)}(x+1 \mid \lambda)-\lambda H_{n}^{(r)}(x \mid \lambda)\right\} .
$$

From (4), we can derive

$$
\begin{aligned}
\sum_{n=0}^{\infty}\left\{H_{n}^{(r)}(x+1 \mid \lambda)-\lambda H_{n}^{(r)}(x \mid \lambda)\right\} \frac{t^{n}}{n !} \\
\quad=\left(\frac{1-\lambda}{e^{t}-\lambda}\right)^{r} e^{(x+1) t}-\lambda\left(\frac{1-\lambda}{e^{t}-\lambda}\right)^{r} e^{x t} \\
\quad=\left(\frac{1-\lambda}{e^{t}-\lambda}\right)^{r} e^{x t}\left(e^{t}-\lambda\right)=(1-\lambda)\left(\frac{1-\lambda}{e^{t}-\lambda}\right)^{r-1} e^{x t} \\
\quad=(1-\lambda) \sum_{n=0}^{\infty} H_{n}^{(r-1)}(x \mid \lambda) \frac{t^{n}}{n !} .
\end{aligned}
$$

By (23) and (24), we get

$$
J_{\lambda}\left(H_{n}^{(r)}(x \mid \lambda)\right)=H_{n}^{(r-1)}(x \mid \lambda) .
$$

From (25), we have

$$
\begin{gathered}
J_{\lambda}^{r}\left(H_{n}^{(r)}(x \mid \lambda)\right)=J_{\lambda}^{r-1}\left(H_{n}^{(r-1)}(x \mid \lambda)\right) \\
=\cdots=H_{n}^{(0)}(x \mid \lambda)=x^{n}, \\
J_{\lambda}^{r}\left(x^{n}\right)=J_{\lambda}^{r} H_{n}^{(0)}(x \mid \lambda)=H_{n}^{(-r)}(x \mid \lambda)=J_{\lambda}^{2 r} H_{n}^{(r)}(x \mid \lambda) .
\end{gathered}
$$

For $s \in \mathbf{Z}_{+}$, from (25), we have

$$
J_{\lambda}^{s}\left(H_{n}^{(r)}(x \mid \lambda)\right)=H_{n}^{(r-s)}(x \mid \lambda) .
$$

On the other hand, by (12), (13), and (25),

$$
\begin{aligned}
J_{\lambda}^{s}\left(H_{n}^{(r)}(x \mid \lambda)\right)= & \left(\frac{e^{t}-\lambda}{1-\lambda}\right)^{s}\left(H_{n}^{(r)}(x \mid \lambda)\right) \\
= & \frac{1}{(1-\lambda)^{s}}\left((1-\lambda)+\sum_{k=1}^{\infty} \frac{t^{k}}{k !}\right)^{s} \\
& \cdot\left(H_{n}^{(r)}(x \mid \lambda)\right) .
\end{aligned}
$$

Thus, by (28), we get

$$
\begin{aligned}
& J_{\lambda}^{s}\left(H_{n}^{(r)}(x \mid \lambda)\right) \\
& =\sum_{m=0}^{s} \frac{\left(\begin{array}{c}
s \\
m
\end{array}\right)}{(1-\lambda)^{m}} \sum_{l=m}^{\infty}\left(\sum_{\substack{k_{1}+\cdots+k_{m}=l \\
k_{j} \geq 1}} \frac{1}{k_{1} ! \cdots k_{m} !}\right) t^{l}\left(H_{n}^{(r)}(x \mid \lambda)\right) \\
& =\sum_{m=0}^{s} \frac{\left(\begin{array}{c}
s \\
m
\end{array}\right)}{(1-\lambda)^{m}} \sum_{l=m}^{\infty} \frac{1}{l !}\left(\sum_{\substack{k_{1}+\cdots+k_{m}=l \\
k_{j} \geq 1}}\left(\begin{array}{c}
l \\
k_{1}, \ldots, k_{m}
\end{array}\right) D^{l}\right) \\
& \cdot H_{n}^{(r)}(x \mid \lambda) \\
& =\sum_{m=0}^{\min \{s, n\}} \frac{\left(\begin{array}{c}
s \\
m
\end{array}\right)}{(1-\lambda)^{m}} \sum_{l=m}^{n}\left(\begin{array}{c}
n \\
l
\end{array}\right) \sum_{\substack{k_{1}+\cdots+k_{m}=l \\
k_{j} \geq 1}}\left(\begin{array}{c}
l \\
k_{1}, \ldots, k_{m}
\end{array}\right) H_{n-l}^{(r)}(x \mid \lambda) \\
& =\sum_{l=0}^{\min \{s, n\}}\left\{\left(\begin{array}{l}
n \\
l
\end{array}\right) \sum_{m=0}^{l} \frac{\left(\begin{array}{c}
s \\
m
\end{array}\right)}{(1-\lambda)^{m}}\right. \\
& \left.\cdot \sum_{\substack{k_{1}+\cdots+k_{m}=l \\
k_{j} \geq 1}}\left(\begin{array}{c}
l \\
k_{1}, \ldots, k_{m}
\end{array}\right)\right\} H_{n-l}^{(r)}(x \mid \lambda)
\end{aligned}
$$

$$
+\sum_{l=\min \{s, n\}+1}^{n}\left\{\left(\begin{array}{l}
n \\
l
\end{array}\right) \sum_{m=0}^{\min \{s, n\}} \frac{\left(\begin{array}{c}
s \\
m
\end{array}\right)}{(1-\lambda)^{m}}\right.
$$

$$
\left.\sum_{\substack{k_{1}+\cdots+k_{m}=l \\
k_{j} \geq 1}}\left(\begin{array}{c}
l \\
k_{1}, \ldots, k_{m}
\end{array}\right)\right\} H_{n-l}^{(r)}(x \mid \lambda) .
$$

Therefore, by (27) and (29), we obtain the following theorem. 
Theorem 2. For any $r, s \geq 0$, one has

$$
\begin{aligned}
& H_{n}^{(r-s)}(x \mid \lambda) \\
& =\sum_{l=0}^{\min \{s, n\}}\left\{\left(\begin{array}{l}
n \\
l
\end{array}\right) \sum_{m=0}^{l} \frac{\left(\begin{array}{c}
s \\
m
\end{array}\right)}{(1-\lambda)^{m}} \sum_{\substack{k_{1}+\cdots+k_{m}=l \\
k_{j} \geq 1}}\left(\begin{array}{c}
l \\
k_{1}, \ldots, k_{m}
\end{array}\right)\right\} \\
& \cdot H_{n-l}^{(r)}(x \mid \lambda) \\
& +\sum_{l=\min \{s, n\}+1}^{n}\left\{\left(\begin{array}{l}
n \\
l
\end{array}\right) \sum_{m=0}^{\min \{s, n\}} \frac{\left(\begin{array}{c}
s \\
m
\end{array}\right)}{(1-\lambda)^{m}}\right. \\
& \left.\cdot \sum_{\substack{k_{1}+\cdots+k_{m}=l \\
k_{j} \geq 1}}\left(\begin{array}{c}
l \\
k_{1}, \ldots, k_{m}
\end{array}\right)\right\} H_{n-l}^{(r)}(x \mid \lambda) .
\end{aligned}
$$

Let us take $s=r-1(r \geq 1)$ in Theorem 2 . Then we obtain the following corollary.

Corollary 3. For $n \geq 0, r \geq 1$, one has

$$
\begin{aligned}
& H_{n}(x \mid \lambda) \\
& =\sum_{l=0}^{\min \{r-1, n\}}\left\{\left(\begin{array}{l}
n \\
l
\end{array}\right) \sum_{m=0}^{l} \frac{\left(\begin{array}{c}
r-1 \\
m
\end{array}\right)}{(1-\lambda)^{m}} \sum_{\substack{k_{1}+\cdots+k_{m}=l \\
k_{j} \geq 1}}\left(\begin{array}{c}
l \\
k_{1}, \ldots, k_{m}
\end{array}\right)\right\} \\
& \cdot H_{n-l}^{(r)}(x \mid \lambda) \\
& +\sum_{l=\min \{r-1, n\}+1}^{n}\left\{\left(\begin{array}{l}
n \\
l
\end{array}\right) \sum_{m=0}^{\min \{r-1, n\}} \frac{\left(\begin{array}{c}
r-1 \\
m
\end{array}\right)}{(1-\lambda)^{m}}\right. \\
& \left.\sum_{\substack{k_{1}+\cdots+k_{m}=l \\
k_{j} \geq 1}}\left(\begin{array}{c}
l \\
k_{1}, \ldots, k_{m}
\end{array}\right)\right\} H_{n-l}^{(r)}(x \mid \lambda) .
\end{aligned}
$$

Let us take $s=r(r \geq 1)$ in Theorem 2. Then we obtain the following corollary.

Corollary 4. For $n \geq 0, r \geq 1$, one has

$$
\begin{aligned}
x^{n}= & \sum_{l=0}^{\min \{r, n\}}\left\{\left(\begin{array}{l}
n \\
l
\end{array}\right) \sum_{m=0}^{l} \frac{\left(\begin{array}{c}
r \\
m
\end{array}\right)}{(1-\lambda)^{m}} \sum_{\substack{k_{1}+\cdots+k_{m}=l \\
k_{j} \geq 1}}\left(\begin{array}{c}
l \\
k_{1}, \ldots, k_{m}
\end{array}\right)\right\} \\
& \cdot H_{n-l}^{(r)}(x \mid \lambda)
\end{aligned}
$$

$$
\begin{aligned}
+\sum_{l=\min \{r, n\}+1}^{n}\left\{\left(\begin{array}{c}
n \\
l
\end{array}\right) \sum_{m=0}^{\min \{r, n\}} \frac{\left(\begin{array}{c}
r \\
m
\end{array}\right)}{(1-\lambda)^{m}}\right. \\
\left.\qquad \sum_{\substack{k_{1}+\cdots+k_{m}=l \\
k_{j} \geq 1}}\left(\begin{array}{c}
l \\
k_{1}, \ldots, k_{m}
\end{array}\right)\right\} H_{n-l}^{(r)}(x \mid \lambda) .
\end{aligned}
$$

Now, we define the analogue of Stirling numbers of the second kind as follows:

$$
S_{\lambda}(n, k)=\frac{1}{k !} \sum_{j=0}^{k}\left(\begin{array}{l}
k \\
j
\end{array}\right)(-\lambda)^{k-j} j^{n}, \quad(n, k \geq 0) .
$$

Note that $S_{1}(n, k)=S(n, k)$ is the Stirling number of the second kind.

From the definition of $\widetilde{\Delta}_{\lambda}$, we have

$$
\widetilde{\Delta}_{\lambda}^{n} f(0)=\sum_{k=0}^{n}\left(\begin{array}{l}
n \\
k
\end{array}\right)(-\lambda)^{n-k} f(k) .
$$

By (33) and (34), we get

$$
S_{\lambda}(n, k)=\frac{1}{k !} \widetilde{\Delta}_{\lambda}^{k} 0^{n}, \quad(n, k \geq 0) .
$$

Let us take $s=2 r$. Then we have

$$
\begin{aligned}
& J_{\lambda}^{r} x^{n} \\
& =H_{n}^{(-r)}(x \mid \lambda) \\
& =\sum_{l=0}^{\min \{2 r, n\}}\left\{\left(\begin{array}{l}
n \\
l
\end{array}\right) \sum_{m=0}^{l} \frac{\left(\begin{array}{c}
2 r \\
m
\end{array}\right)}{(1-\lambda)^{m}} \sum_{\substack{k_{1}+\cdots+k_{m}=l \\
k_{j} \geq 1}}\left(\begin{array}{c}
l \\
k_{1}, \ldots, k_{m}
\end{array}\right)\right\} \\
& \cdot H_{n-l}^{(r)}(x \mid \lambda) \\
& +\sum_{l=\min \{2 r, n\}+1}^{n}\left\{\begin{array}{c}
n \\
l
\end{array}\right)^{\min \{2 r, n\}} \frac{\left(\begin{array}{c}
2 r \\
m
\end{array}\right)}{(1-\lambda)^{m}} \\
& \quad=\frac{1}{(1-\lambda)^{r}} \sum_{j=0}^{r}\left(\begin{array}{c}
r \\
j
\end{array}\right)^{(-\lambda)^{r-j}(x+j)^{n} .} \\
& \left.J_{\lambda}^{r} x^{n}=\left(\frac{1}{1-\lambda} \tilde{\Delta}_{\lambda}\right)^{r} x^{n}\left(\begin{array}{c}
l \\
k_{1}+\cdots+k_{m}=l \\
k_{j} \geq 1
\end{array}\right)\right\} H_{n-l}^{(r)}(x \mid \lambda),
\end{aligned}
$$


By (36), we get

$$
\begin{aligned}
& \frac{1}{(1-\lambda)^{r}} \sum_{j=0}^{r}\left(\begin{array}{l}
r \\
j
\end{array}\right)(-\lambda)^{r-j}(x+j)^{n} \\
& =\frac{1}{(1-\lambda)^{r}} \widetilde{\Delta}_{\lambda}^{r} x^{n} \\
& =\sum_{l=0}^{\min \{2 r, n\}}\left\{\left(\begin{array}{l}
n \\
l
\end{array}\right) \sum_{m=0}^{l} \frac{\left(\begin{array}{c}
2 r \\
m
\end{array}\right)}{(1-\lambda)^{m}} \sum_{\substack{k_{1}+\cdots+k_{m}=l \\
k_{j} \geq 1}}\left(\begin{array}{c}
l \\
k_{1}, \ldots, k_{m}
\end{array}\right)\right\} \\
& \cdot H_{n-l}^{(r)}(x \mid \lambda) \\
& +\sum_{l=\min \{2 r, n\}+1}^{n}\left\{\left(\begin{array}{l}
n \\
l
\end{array}\right) \sum_{m=0}^{\min \{2 r, n\}} \frac{\left(\begin{array}{c}
2 r \\
m
\end{array}\right)}{(1-\lambda)^{m}}\right. \\
& \left.\cdot \sum_{\substack{k_{1}+\cdots+k_{m}=l \\
k_{j} \geq 1}}\left(\begin{array}{c}
l \\
k_{1}, \ldots, k_{m}
\end{array}\right)\right\} H_{n-l}^{(r)}(x \mid \lambda) .
\end{aligned}
$$

Let us take $x=0$ in (37). Then we obtain the following theorem.

Theorem 5. We have

$$
\begin{aligned}
& \frac{r !}{(1-\lambda)^{r}} S_{\lambda}(n, r) \\
& =\frac{r !}{(1-\lambda)^{r}} \frac{\widetilde{\Delta}_{\lambda}^{r} 0^{n}}{r !} \\
& =\sum_{l=0}^{\min \{2 r, n\}}\left\{\left(\begin{array}{l}
n \\
l
\end{array}\right) \sum_{m=0}^{l} \frac{\left(\begin{array}{c}
2 r \\
m
\end{array}\right)}{(1-\lambda)^{m}} \sum_{\substack{k_{1}+\cdots+k_{m}=l \\
k_{j} \geq 1}}\left(\begin{array}{c}
l \\
k_{1}, \ldots, k_{m}
\end{array}\right)\right\} \\
& \cdot H_{n-l}^{(r)}(\lambda) \\
& +\sum_{l=\min \{2 r, n\}+1}^{n}\left\{\left(\begin{array}{l}
n \\
l
\end{array}\right) \sum_{m=0}^{\min \{2 r, n\}} \frac{\left(\begin{array}{c}
2 r \\
m
\end{array}\right)}{(1-\lambda)^{m}}\right. \\
& \left.\cdot \sum_{\substack{k_{1}+\cdots+k_{m}=l \\
k_{j} \geq 1}}\left(\begin{array}{c}
l \\
k_{1}, \ldots, k_{m}
\end{array}\right)\right\} H_{n-l}^{(r)}(\lambda) \\
& =\sum_{m=0}^{\min \{r, n\}} \frac{\left(\begin{array}{c}
r \\
m
\end{array}\right)}{(1-\lambda)^{m}} \sum_{\substack{k_{1}+\cdots+k_{m}=n \\
k_{j} \geq 1}}\left(\begin{array}{c}
n \\
k_{1}, \ldots, k_{m}
\end{array}\right) .
\end{aligned}
$$

Let us consider $s=2 r-1$ in the identity of Theorem 2 . Then we have

$$
\begin{aligned}
& J_{\lambda}^{r-1} x^{n} \\
& =H_{n}^{-(r-1)}(x \mid \lambda) \\
& =\sum_{l=0}^{\min \{2 r-1, n\}}\left\{\left(\begin{array}{l}
n \\
l
\end{array}\right) \sum_{m=0}^{l} \frac{\left(\begin{array}{c}
2 r-1 \\
m
\end{array}\right)}{(1-\lambda)^{m}} \sum_{\substack{k_{1}+\cdots+k_{m}=l \\
k_{j} \geq 1}}\left(\begin{array}{c}
l \\
k_{1}, \ldots, k_{m}
\end{array}\right)\right\} \\
& \cdot H_{n-l}^{(r)}(x \mid \lambda) \\
& +\sum_{l=\min \{2 r-1, n\}+1}^{n}\left\{\left(\begin{array}{l}
n \\
l
\end{array}\right) \sum_{m=0}^{\min \{2 r-1, n\}} \frac{\left(\begin{array}{c}
2 r-1 \\
m
\end{array}\right)}{(1-\lambda)^{m}}\right. \\
& \left.\cdot \sum_{\substack{k_{1}+\cdots+k_{m}=l \\
k_{j} \geq 1}}\left(\begin{array}{c}
l \\
k_{1}, \ldots, k_{m}
\end{array}\right)\right\} H_{n-l}^{(r)}(x \mid \lambda) \\
& =\frac{1}{(1-\lambda)^{r-1}} \sum_{j=0}^{r-1}\left(\begin{array}{c}
r-1 \\
j
\end{array}\right)(-\lambda)^{r-1-j}(x+j)^{n} \\
& =\frac{1}{(1-\lambda)^{r-1}} \widetilde{\Delta}_{\lambda}^{r-1} x^{n} \text {. }
\end{aligned}
$$

Let us take $x=0$ in (39). Then we obtain the following theorem.

Theorem 6. For $n \geq 0$ and $r \geq 1$, one has

$$
\begin{aligned}
& \frac{(r-1) !}{(1-\lambda)^{r-1}} S_{\lambda}(n, r-1) \\
& =\frac{(r-1) !}{(1-\lambda)^{r-1}} \frac{\widetilde{\Delta}_{\lambda}^{r-1} 0^{n}}{(r-1) !} \\
& =\sum_{l=0}^{\min \{2 r-1, n\}}\left\{\left(\begin{array}{l}
n \\
l
\end{array}\right) \sum_{m=0}^{l} \frac{\left(\begin{array}{c}
2 r-1 \\
m
\end{array}\right)}{(1-\lambda)^{m}} \sum_{\substack{k_{1}+\cdots+k_{m}=l \\
k_{j} \geq 1}}\left(\begin{array}{c}
l \\
k_{1}, \ldots, k_{m}
\end{array}\right)\right\} \\
& \text { - } H_{n-l}^{(r)}(\lambda) \\
& +\sum_{l=\min \{2 r-1, n\}+1}^{n}\left\{\left(\begin{array}{l}
n \\
l
\end{array}\right) \sum_{m=0}^{\min \{2 r-1, n\}} \frac{\left(\begin{array}{c}
2 r-1 \\
m
\end{array}\right)}{(1-\lambda)^{m}}\right. \\
& \left.\cdot \sum_{\substack{k_{1}+\cdots+k_{m}=l \\
k_{j} \geq 1}}\left(\begin{array}{c}
l \\
k_{1}, \ldots, k_{m}
\end{array}\right)\right\} H_{n-l}^{(r)}(\lambda) \text {. }
\end{aligned}
$$


Remark 7. Note that

$$
\begin{aligned}
& \frac{(r-1) !}{(1-\lambda)^{r-1}} S_{\lambda}(n, r-1) \\
& =\sum_{l=0}^{\min \{r, n\}}\left\{\left(\begin{array}{l}
n \\
l
\end{array}\right) \sum_{m=0}^{l} \frac{\left(\begin{array}{c}
r \\
m
\end{array}\right)}{(1-\lambda)^{m}} \sum_{\substack{k_{1}+\cdots+k_{m}=l \\
k_{j} \geq 1}}\left(\begin{array}{c}
l \\
k_{1}, \ldots, k_{m}
\end{array}\right)\right\} \\
& \text { - } H_{n-l}(\lambda) \\
& +\sum_{l=\min \{r, n\}+1}^{n}\left\{\left(\begin{array}{l}
n \\
l
\end{array}\right) \sum_{m=0}^{\min \{r, n\}} \frac{\left(\begin{array}{c}
r \\
m
\end{array}\right)}{(1-\lambda)^{m}}\right. \\
& \left.\cdot \sum_{\substack{k_{1}+\cdots+k_{m}=l \\
k_{j} \geq 1}}\left(\begin{array}{c}
l \\
k_{1}, \ldots, k_{m}
\end{array}\right)\right\} H_{n-l}(\lambda)
\end{aligned}
$$

\section{Acknowledgment}

The authors would like to express their gratitude to the referees for their valuable suggestions.

\section{References}

[1] T. Kim, "Identities involving Frobenius-Euler polynomials arising from non-linear differential equations," Journal of Number Theory, vol. 132, no. 12, pp. 2854-2865, 2012.

[2] T. Kim and J. Choi, "A note on the product of Frobenius-Euler polynomials arising from the $p$-adic integral on $Z_{p}$," Advanced Studies in Contemporary Mathematics, vol. 22, no. 2, pp. 215223, 2012.

[3] S. Roman, The Umbral Calculus, Dover, New York, NY, USA, 2005.

[4] Y. Simsek, O. Yurekli, and V. Kurt, "On interpolation functions of the twisted generalized Frobenius-Euler numbers," Advanced Studies in Contemporary Mathematics, vol. 15, no. 2, pp. 187-194, 2007.

[5] K. Shiratani, "On Euler numbers," Memoirs of the Faculty of Science. Kyushu University A, vol. 27, pp. 1-5, 1973.

[6] S. Araci and M. Acikgoz, "A note on the frobenius-euler numbers and polynomials associated with bernstein polynomials," Advanced Studies in Contemporary Mathematics, vol. 22, no. 3, pp. 399-406, 2012.

[7] L. Carlitz, "Some polynomials related to the Bernoulli and Euler polynomials," Utilitas Mathematica, vol. 19, pp. 81-127, 1981.

[8] M. Can, M. Cenkci, V. Kurt, and Y. Simsek, "Twisted Dedekind type sums associated with Barnes' type multiple FrobeniusEuler l-functions," Advanced Studies in Contemporary Mathematics, vol. 18, no. 2, pp. 135-160, 2009.

[9] I. N. Cangul, V. Kurt, H. Ozden, and Y. Simsek, "On the higher-order $w$-q-Genocchi numbers," Advanced Studies in Contemporary Mathematics, vol. 19, no. 1, pp. 39-57, 2009.

[10] R. Dere and Y. Simsek, "Applications of umbral algebra to some special polynomials," Advanced Studies in Contemporary Mathematics, vol. 22, no. 3, pp. 433-438, 2012.
[11] K. Shiratani and S. Yamamoto, "On a $p$-adic interpolation function for the Euler numbers and its derivatives," Memoirs of the Faculty of Science. Kyushu University A, vol. 39, no. 1, pp. 113125, 1985.

[12] D. S. Kim and T. Kim, "Some identities of Frobenius-Euler polynomials arising from umbral calculus," Advances in Difference Equations, vol. 2012, article 196, 2012. 


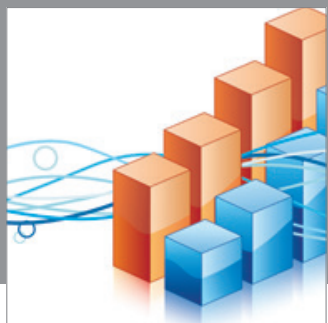

Advances in

Operations Research

mansans

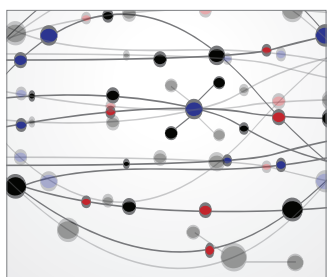

The Scientific World Journal
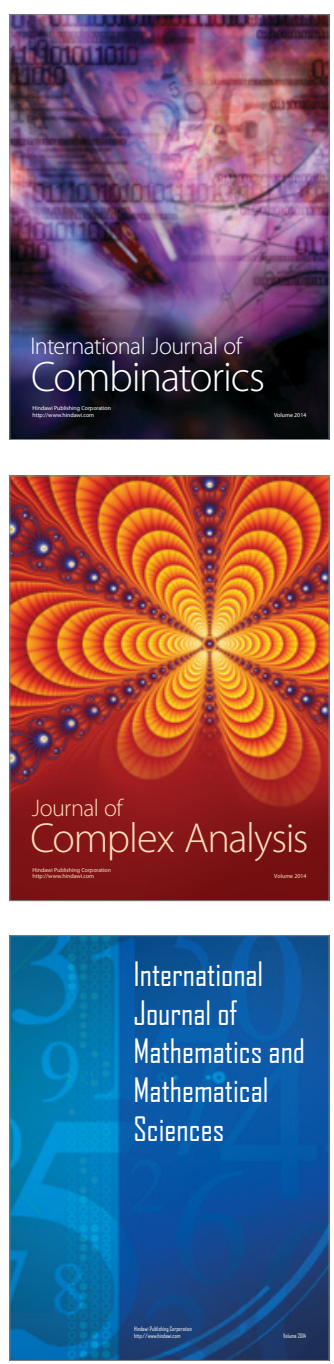
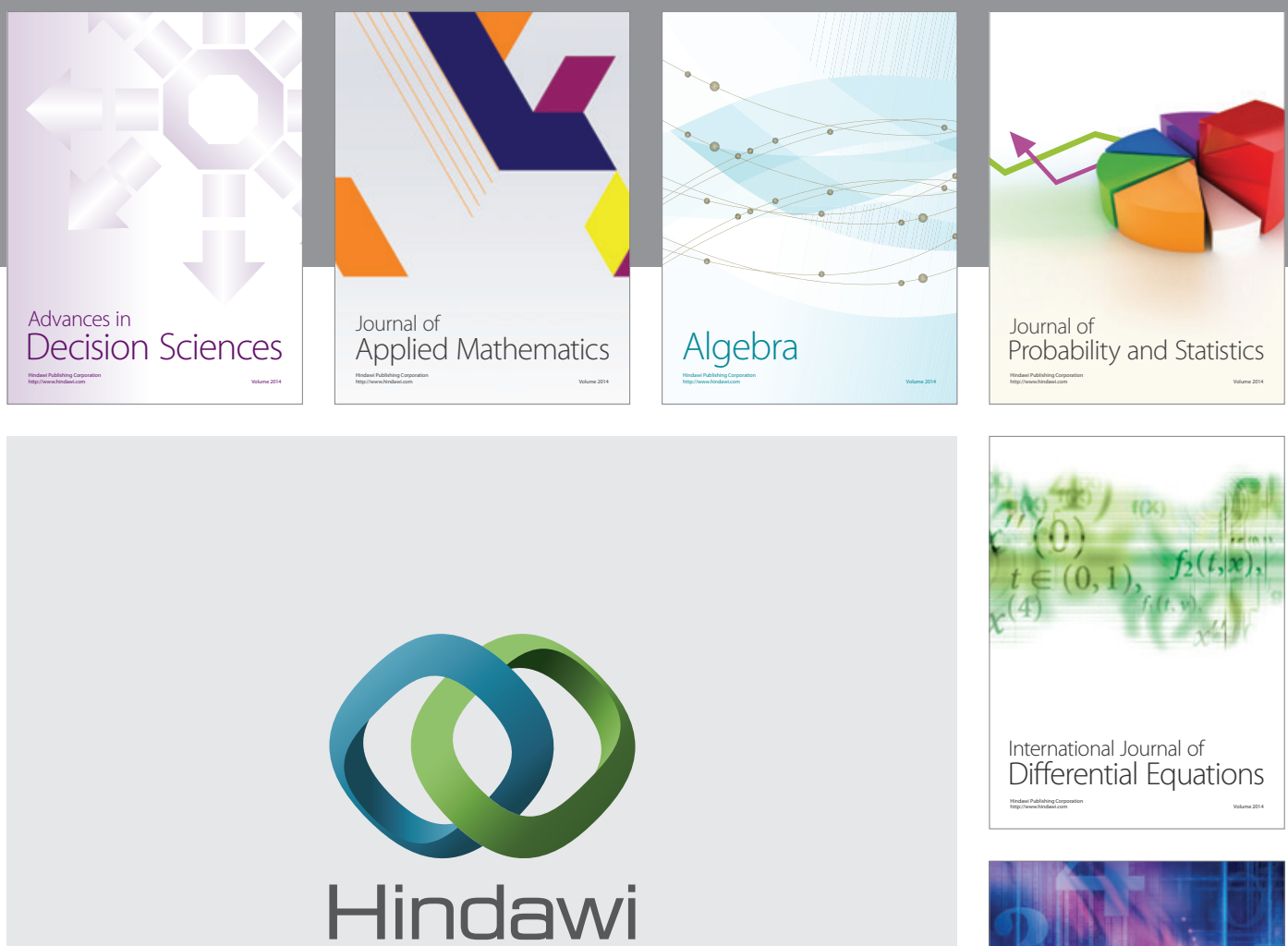

Submit your manuscripts at http://www.hindawi.com
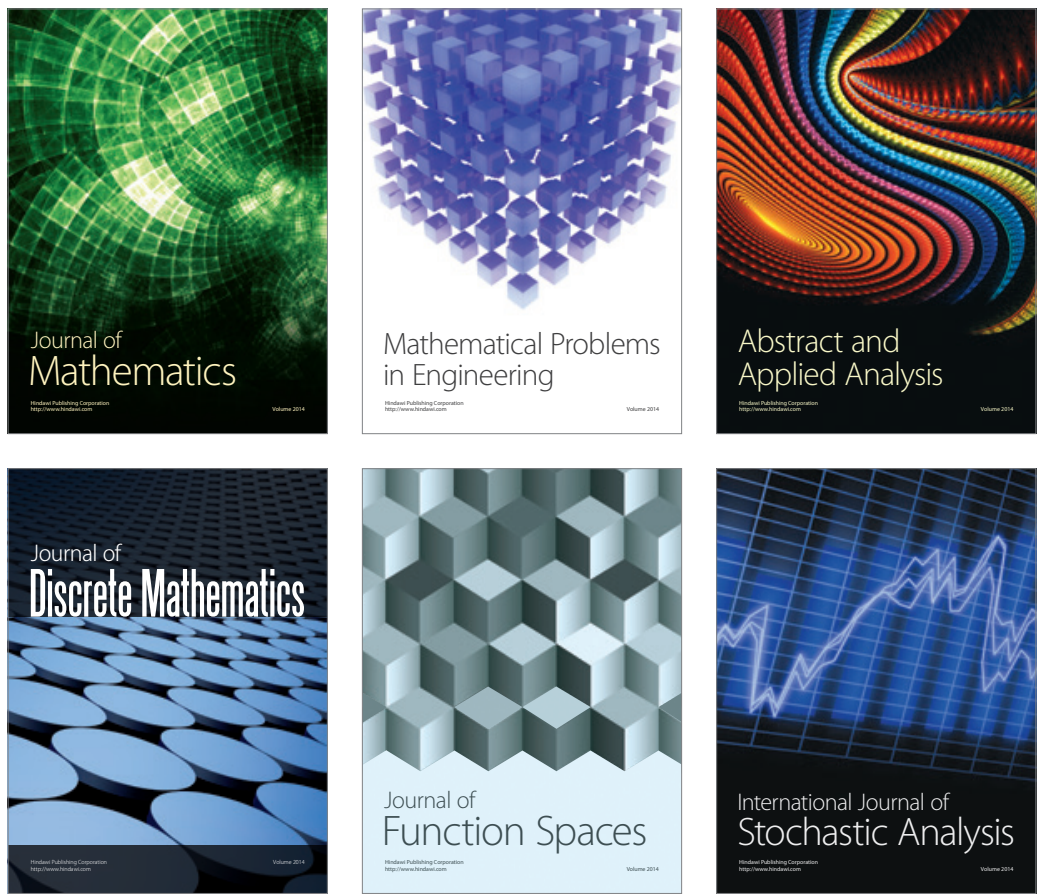

Journal of

Function Spaces

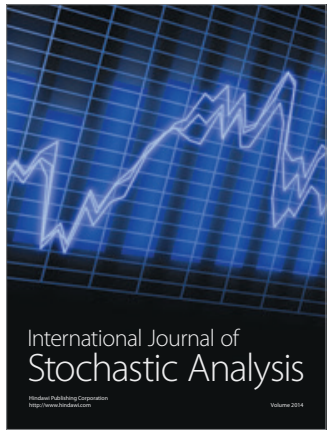

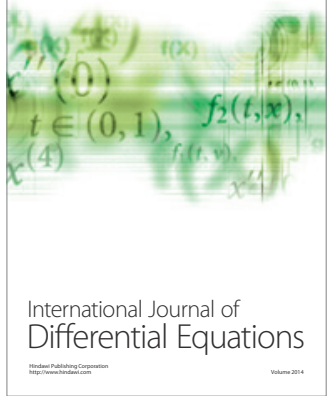
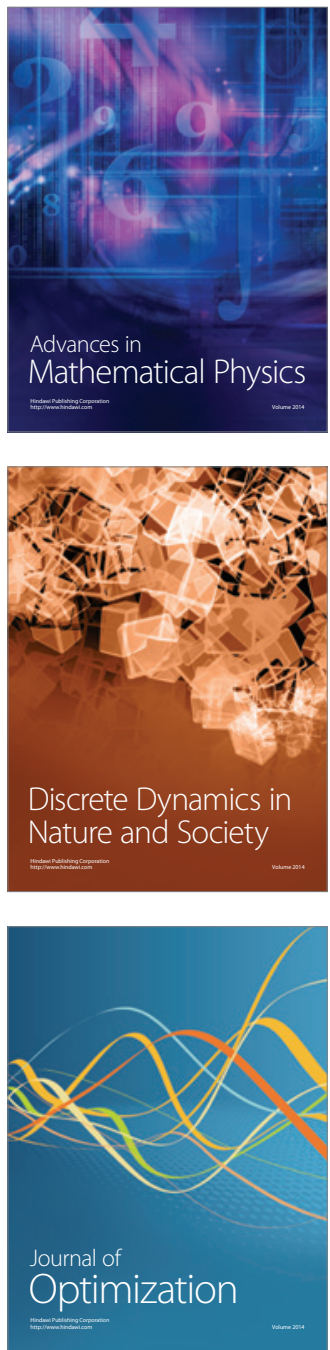